Bull. Austral. Math. Soc.

VOL. $72(2005)$ [391-402]

\title{
EXAMPLES AND CLASSIFICATION OF RIEMANNIAN SUBMERSIONS SATISFYING A BASIC EQUALITY
}

\author{
BANG-Yen Chen
}

\begin{abstract}
In an earlier article we obtain a sharp inequality for an arbitrary isometric immersion from a Riemannian manifold admitting a Riemannian submersion with totally geodesic fibres into a unit sphere. In this article we investigate the immersions which satisfy the equality case of the inequality. As a by-product, we discover a new characterisation of Cartan hypersurface in $S^{4}$.
\end{abstract}

\section{InTRODUCTION.}

Let $M$ and $B$ be Riemannian manifolds. A Riemannian submersion $\pi: M \rightarrow B$ is a mapping of $M$ onto $B$ satisfying the following two axioms:

(S1) $\pi$ has maximal rank;

(S2) the differential $\pi_{*}$ preserves lengths of horizontal vectors.

The mappings between Riemannian manifolds satisfying these two axioms were studied by Nagano in [10] in terms of fibred Riemannian manifolds. In particular, he derived the fundamental equations analogous to Weingarten's formulas for Riemannian submanifolds. O'Neill further studied such mappings in [11] and called such mappings Riemannian submersions.

Throughout this article, we only consider Riemannian submersions $\pi: M \rightarrow B$ with $m>b>0$, where $m=\operatorname{dim} M$ and $b=\operatorname{dim} B$.

For each $p \in B, \pi^{-1}(p)$ is an $(m-b)$-dimensional submanifold of $M$. The submanifolds $\pi^{-1}(p), p \in B$, are called fibres. A vector field on $M$ is called vertical if it is always tangent to fibres; and horizontal if it is always orthogonal to fibres. We use corresponding terminology for individual tangent vectors as well. A vector field on $M$ is called basic if $X$ is horizontal and $\pi$-related to a vector field $X_{*}$ on $B$, that is, $\pi_{*} X_{u}=X_{* \pi(u)}$, for all $u \in M$.

Let $\mathcal{H}$ and $\mathcal{V}$ denote the projections of tangent spaces of $M$, onto the subspaces of horizontal and vertical vectors, respectively. We use the same letters to denote the horizontal and vertical distributions.

Received 4th July, 2005

Copyright Clearance Centre, Inc. Serial-fee code: 0004-9727/05 $\$ A 2.00+0.00$. 
Associated with a Riemannian submersion $\pi: M \rightarrow B$, there are two natural $(1,2)$ tensors $T$ and $A$ on $M$ defined as follows:

For vector fields $E, F$ tangent to $M$, the tensor $T$ is defined by (see, for instance, [11]):

$$
T_{E} F=\mathcal{H} \nabla_{\mathcal{V E}}(\mathcal{V} F)+\mathcal{V} \nabla_{\mathcal{H} E}(\mathcal{H} F)
$$

The other tensor $A$, known as the integrability tensor, is defined by

$$
A_{E} F=\mathcal{V} \nabla_{\mathcal{H E}}(\mathcal{H} F)+\mathcal{H} \nabla_{\mathcal{H E}}(\mathcal{V} F) .
$$

In particular, for any horizontal vector field $X$ and any vertical vector field $V$, we have

$$
A_{X} V=\mathcal{H} \nabla_{X} V .
$$

It is well-known that, for horizontal vector fields $X, Y$, the tensor $A$ has the alternation property:

$$
A_{X} Y=-A_{Y} X
$$

Associated with each Riemannian submersion $\pi: M \rightarrow B$, the invariant $\breve{A}_{\pi}$ on $M$ is defined by

$$
\breve{A}_{\pi}=\sum_{i=1}^{b} \sum_{s=b+1}^{m}\left\|A_{X_{i}} V_{s}\right\|^{2}, \quad b=\operatorname{dim} B, m=\operatorname{dim} M,
$$

where $X_{1}, \ldots, X_{b}$ are orthonormal basic horizontal vector fields and $V_{b+1}, \ldots, V_{m}$ are orthonormal vertical vector fields on $M$. It is easy to verify that $\breve{A}_{\pi}$ is well-defined. It is easy to see that a Riemannian submersion $\pi: M \rightarrow B$ has totally geodesic fibres if and only if $T$ vanishes identically.

By applying an idea from $[4,5]$, the following inequality was established in [6].

THEOREM A. Let $\pi: M \rightarrow B$ be a Riemannian submersion with totally geodesic fibres. Then, for any isometric immersion of $M$ into a unit sphere $S^{N}$, we have

$$
\breve{A}_{\pi} \leqslant \frac{m^{2}}{4} H^{2}+b(m-b)
$$

where $b=\operatorname{dim} B, m=\operatorname{dim} M$ and $H^{2}$ is the squared mean curvature of the immersion.

By applying this theorem the author proves that if a Riemannian manifold $M$ admits a non-trivial Riemannian submersion $\pi: M \rightarrow B$ with totally geodesic fibres, then it cannot be isometrically immersed in any Riemannian manifold of non-positively sectional curvature as a minimal submanifold. Moreover, he proves that if $M$ admits a minimal immersion into a Euclidean space, then locally $M$ is the Riemannian product $F \times B$ 
of a fibre $F$ and the base manifold $B$ and the minimal immersion of $M$ is locally the product immersion $\left(\phi_{F}, \phi_{B}\right)$ of some minimal isometric immersions $\phi_{F}: F \rightarrow \mathbb{E}^{m_{1}}$ and $\phi_{B}: B \rightarrow \mathbb{E}^{m_{2}}$ into some Euclidean spaces (see [6] for details). Here, by a non-trivial Riemannian submersion we mean a Riemannian submersion whose horizontal and vertical distributions are not both totally geodesic distributions.

In this article we investigate the Riemannian submersions $\pi: M \rightarrow B$ with totally geodesic fibres and the isometric immersions of $M$ in $S^{N}$ which satisfy the equality case of inequality (1.6) identically. In order to do so, we recall briefly the definition of Hopf's fibration:

Consider $S^{2 n+1}$ as the unit hypersphere in $\mathbb{C}^{n+1}$ centred at the origin and let $z$ be its unit outward normal. Let $\widetilde{J}$ be the natural almost complex structure on $\mathbb{C}^{n+1}$. Then $\widetilde{J} z$ defines an integrable distribution on $S^{2 n+1}$ with totally geodesic leaves. Identifying the leaves as points we obtain the complex projective $n$-space $C P^{n}$. By taking as the horizontal distribution, the orthogonal complements to $\widetilde{J}_{z}$ in $T S^{2 n+1}$, one can make this into a Riemannian submersion, known as the Hopf fibration:

$$
\pi_{C}: S^{2 n+1} \rightarrow C P^{n}
$$

with great circles as fibres.

Similarly, consider $S^{4 k+3}$ as the unit hypersphere in $\mathbb{Q}^{k+1}$ and let $z$ be its unit outward normal. Let $J_{1}, J_{2}, J_{3}$ be the natural almost complex structures on $\mathbb{Q}^{k+1}$ with

$$
J_{1} J_{2}=J_{3}, \quad J_{2} J_{3}=J_{1}, \quad J_{3} J_{1}=J_{2} .
$$

Then $J_{1} z, J_{2} z, J_{3} z$ define an integrable distribution on $S^{4 k+3}$ with totally geodesic leaves. Identifying the leaves as points we obtain the quaternionic projective $k$-space $Q P^{k}$ which can be made into a Riemannian submersion:

$$
\pi_{Q}: S^{4 k+3} \rightarrow Q P^{k}
$$

by taking as the horizontal distribution, the orthogonal complements to $J_{1} z, J_{2} z, J_{3} z$ in $T S^{4 k+3}$. Fibres of $\pi_{Q}$ are totally geodesic 3-spheres in $S^{4 k+3}$. The projection (1.9) is also known as the Hopf fibration.

The following result shows that there exist many Riemannian manifolds which admit Riemannian submersions with totally geodesic fibres and which also admit isometric immersions satisfying the equality case of (1.6) identically into some unit spheres.

THEOREM 1. We have:

(a) Let $B$ be a Kaehler submanifold of $C P^{n}$ and let $\pi_{C}^{B}: \pi_{C}^{-1}(B) \rightarrow B$ be the restriction of Hopf's fibration $\pi_{C}$ to $\pi_{C}^{-1}(B) \subset S^{2 n+1}$. Then the inclusion map $\iota_{C}: \pi_{C}^{-1}(B) \rightarrow S^{2 n+1}$ is an isometric immersion such that

(a.1) the fibres of $\pi_{C}^{B}$ are fibres of the Hopf fibration $\pi_{C}$ and 
(a.2) $\iota_{C}$ satisfies the equality case of (1.6) identically (with $m=1+b$; $\left.b=\operatorname{dim}_{\mathbb{R}} B\right)$.

(b) Let $B$ be an open portion of a totally geodesic $Q P^{\ell} \subset Q P^{k}$ and let $\pi_{Q}^{B}$ : $\pi_{Q}^{-1}(B) \rightarrow B$ be the restriction of Hopf's fibration $\pi_{Q}$ to $\pi_{Q}^{-1}(B) \subset S^{4 k+3}$. Then the inclusion map $\iota_{Q}: \pi_{Q}^{-1}(B) \rightarrow S^{4 k+3}$ is an isometric immersion such that

(b.1) the fibres of $\pi_{Q}^{B}$ are fibres of Hopf's fibration $\pi_{Q}$ and

(b.2) $\iota_{Q}$ satisfies the equality case of (1.6) identically (with $m=3+b$; $\left.b=\operatorname{dim}_{\mathbb{R}} B\right)$.

Let $\pi: M \rightarrow B$ and $\pi^{\prime}: M^{\prime} \rightarrow B^{\prime}$ be two Riemannian submersions with totally geodesic fibres. Then $\pi$ and $\pi^{\prime}$ are said to be equivalent provided there exists an isometry $f: M \rightarrow M^{\prime}$ which induces an isometry $f_{B}: B \rightarrow B^{\prime}$ so that the following diagram commutes (see $[7,8]$ ):

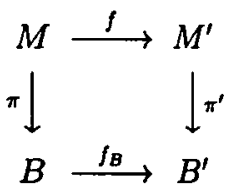

As a converse to Theorem 1 , we have the following.

THEOREM 2. Let $\pi: M \rightarrow B$ be a Riemannian submersion with totally geodesic fibres. Then we have:

(i) If $M$ admits an isometric imbedding $\phi: M \rightarrow S^{2 n+1}$ which carries fibres of $\pi$ to fibres of $\pi_{C}: S^{2 n+1} \rightarrow C P^{n}$ and $\phi$ satisfies the equality sign of (1.6), then there exists a Kaehler submanifold $B_{1} \subset C P^{n}$ such that $\pi: M \rightarrow B$ is equivalent to $\pi_{C}: \pi_{C}^{-1}\left(B_{1}\right) \rightarrow B_{1}$ and $\phi$ is congruent to the inclusion map: $\iota_{C}: \pi_{C}^{-1}\left(B_{1}\right) \rightarrow S^{2 n+1}$.

(ii) If $M$ admits an isometric imbedding $\phi: M \rightarrow S^{4 k+3}$ which carries fibres of $\pi$ to fibres of $\pi_{Q}: S^{4 k+3} \rightarrow Q P^{k}$ and $\phi$ satisfies the equality sign of (1.6), then there exists an open portion $B_{2}$ of some totally geodesic $Q P^{\ell} \subset Q P^{k}$ such that $\pi: M \rightarrow B$ is equivalent to $\pi_{Q}: \pi_{Q}^{-1}\left(B_{2}\right) \rightarrow B_{2}$ and $\phi$ is congruent to the inclusion map: $\iota_{Q}: \pi_{Q}^{-1}\left(B_{2}\right) \rightarrow S^{4 k+3}$.

Let $\pi: M \rightarrow B$ be a Riemannian submersion. An isometric immersion $\phi: M \rightarrow S^{N}$ is called mixed-totally geodesic if its second fundamental form $h$ satisfies

$$
h(X, V)=0
$$

for any horizontal vector $X$ and vertical vector $V$ on $M$.

In view of Theorem 2 , we prove the following.

THEOREM 3. Let $\pi: M \rightarrow B$ be a Riemannian submersion with totally geodesic fibres. If an isometric immersion $\phi: M \rightarrow S^{N}$ carries the fibres of $\pi$ to totally geodesic submanifolds of $S^{N}$ and satisfies the equality case of (1.6), then we have 
(1) $\operatorname{dim} M \geqslant 3$ and

(2) $M$ is immersed as a minimal mixed-totally geodesic submanifold of $S^{N}$.

The Cartan hypersurface in $S^{4} \subset \mathbb{E}^{5}$ is defined by the equation:

$$
2 x_{5}^{3}+3\left(x_{1}^{2}+x_{2}^{2}\right) x_{5}-6\left(x_{3}^{2}+x_{4}^{2}\right) x_{5}+3 \sqrt{3}\left(x_{1}^{2}-x_{2}^{2}\right) x_{4}+6 \sqrt{3} x_{1} x_{2} x_{3}=2 .
$$

É. Cartan proved that this hypersurface is the homogeneous Riemannian manifold $S U(3) / S O(3)$ (equipped with a suitable metric) and its principal curvatures in $S^{4}$ are given by $0, \sqrt{3},-\sqrt{3}$. It is also known that the Cartan hypersurface is a tubular hypersurface about the Veronese surface with radius $r=\pi / 2$.

The next result classifies isometric immersions $\phi: M^{3} \rightarrow S^{4}$ from Riemannian 3manifold into $S^{4}$ which satisfy the hypothesis of Theorem 3 . This result provides a new characterisation of Cartan's hypersurface.

TheOREM 4. Let $\pi: M^{3} \rightarrow B$ be a Riemannian submersion with totally geodesic fibres. If $\phi: M^{3} \rightarrow S^{4}$ is an isometric immersion carrying fibres to totally geodesic submanifolds of $S^{4}$ and if $\phi$ satisfies the equality case of (1.6), then either

(a) $\phi$ is a totally geodesic hypersurface of $S^{4}$ or

(b) $\phi$ is congruent to the Cartan hypersurface.

\section{PROOF OF THEOREM 1.}

Let $B$ be a submanifold of $C P^{n}$ and $\pi_{C}^{B}: \pi_{C}^{-1}(B) \rightarrow B$ the Hopf fibration restricted to $\pi_{C}^{-1}(B)$. Consider the unit sphere $S^{2 n+1}$ as the unit hypersphere of the complex Euclidean $(n+1)$-space $\mathbb{C}^{n+1}$. Let $z$ be the unit outward normal of $S^{2 n+1}$ and $\tilde{J}$ the almost complex structure of $\mathbb{C}^{n+1}$. Then fibres of $\pi_{C}^{B}$ are great circles in $S^{2 n+1}$ and the characteristic vector field $\xi$ on the Sasakian $S^{2 n+1}$ is given by $\xi=\widetilde{J} z$. Clearly, the restriction of $\xi$ to $\pi_{C}^{-1}(B)$ is a vertical vector field of the Riemannian submersion $\pi_{C}^{B}: \pi_{1}^{-1}(B) \rightarrow B$ and the fibres of $\pi$ are fibres of the Hopf fibration $\pi_{C}: S^{2 n+1} \rightarrow C P^{n}$.

Let $\widetilde{\nabla}^{\mathbb{C}}$ and $\nabla$ denote the Levi-Civita connections on $\mathbb{C}^{n+1}$ and $\pi_{C}^{-1}(B)$, respectively. Then we have

$$
\tilde{\nabla}_{X}^{c}(\tilde{J} z)=\tilde{J} X
$$

for any horizontal vector $X$ of $\pi_{C}^{B}: \pi_{C}^{-1}(B) \rightarrow B$.

For any horizontal vector $E$, we have $0=\langle E, z\rangle=\langle E, \tilde{J} z\rangle=0$. Thus we get

$$
0=\langle\tilde{J} E, z\rangle=\langle\tilde{J} E, \tilde{J} z\rangle=0
$$

which implies that the tangential component, $P E$, of $\widetilde{J} E$ is also a horizontal vector on $\pi_{C}^{-1}(B)$. Hence, we find from (1.3) and (2.1) that

$$
A_{X} \xi=P X \text {. }
$$


Consequently, we obtain from (1.5) that

$$
\breve{A}_{\pi}=\left\|P_{\mathcal{H}}\right\|^{2}
$$

where $P_{\mathcal{H}}$ is the endomorphism of the horizontal distribution $\mathcal{H}$ defined to be the restriction of $P$ on $\mathcal{H}$.

Now, suppose that $B$ is a Kaehler submanifold of $C P^{n}$. Then $B$ is a minimal submanifold of $C P^{n}$. Thus, according to a result of [9], the pre-image $\pi_{C}^{-1}(B)$ is a minimal submanifold of $S^{2 n+1}$.

Because the horizontal distribution $\mathcal{H}$ is $\widetilde{J}$-invariant, (2.3) implies that $\breve{A}_{\pi}$ $=\operatorname{dim}_{\mathbb{R}} B=b$. From these we conclude that the inclusion map $\iota_{C}: \pi_{C}^{-1}(B) \rightarrow S^{2 n+1}$ satisfies the equality case of (1.6) identically. This proves statement (a).

Next, assume that $B$ is an open portion of a totally geodesic $Q P^{\ell} \subset Q P^{k}$. Consider the unit sphere $S^{4 k+3}$ as the unit hypersphere in the flat quaternionic $(m+1)$-space $\mathbb{Q}^{k+1}$ defined by $\left\{z \in Q^{k+1}:|z|=1\right\}$. Let $z$ be the unit outward normal of $S^{4 k+3}$ in $\mathbb{Q}^{k+1}$ and let $J_{1}, J_{2}, J_{3}$ be the standard quaternionic structures on $\mathbb{Q}^{m+1}$.

If we put $\xi_{\alpha}=J_{\alpha} z$, then the fibres of $\pi_{Q}^{B}: \pi_{Q}^{-1}(B) \rightarrow B$ are totally geodesic 3-spheres in $S^{4 k+3}$. Moreover, $\xi_{1}, \xi_{2}, \xi_{3}$ form an orthonormal frame of the vertical distribution $\mathcal{V}$ of the Riemannian submersion $\pi_{Q}^{B}$.

Let $\widetilde{\nabla}^{Q}$ and $\nabla$ denote the Levi-Civita connection on $\mathbb{Q}^{k+1}$ and $\pi_{Q}^{-1}(B)$, respectively. Then, for any horizontal vector $X$ on $\pi_{Q}^{-1}(B)$, we have

$$
\widetilde{\nabla}_{X}\left(J_{\alpha} z\right)=J_{\alpha} X, \alpha=1,2,3
$$

Since $B$ is an open portion of a totally geodesic $Q P^{\ell}$, we find from (1.3) and (2.4) that

$$
A_{X} \xi_{\alpha}=\mathcal{H}\left(J_{\alpha} X\right)=J_{\alpha} X, \quad \alpha=1,2,3,
$$

for any horizontal vector field $X$. From (2.5) we obtain

$$
\breve{A}_{\pi_{Q}^{B}}=12 \ell=3 \operatorname{dim}_{\mathbf{R}} B \text {. }
$$

Since $B$ is totally gedoesic in $Q P^{k}$, the inclusion map $\iota_{Q}: \pi_{2}^{-1}(B) \rightarrow S^{4 k+3}$ is also totally geodesic. In particular, we have $H=0$. Hence, we may conclude from (2.6) that the equality case of (1.6) holds identically (with $b=4 \ell$ and $m=3+b$ ). This proves statement (b).

\section{Proof of Theorem 2.}

Assume that $\pi: M \rightarrow B$ is a Riemannian submersion with totally geodesic fibres. Suppose that $M$ admits an isometric imbedding $\phi: M \rightarrow S^{2 n+1} \subset \mathbb{C}^{n+1}$ which carries the fibres of $\pi$ to fibres of the Hopf fibration $\pi_{C}: S^{2 n+1} \rightarrow C P^{n}$. 
Let $z$ denote the position vector field of $M$ in $\mathbb{C}^{2 m+1}$. Then $\tilde{J}_{z}$ is a unit vertical vector field on $M$. For any horizontal vector $X$ of $M$, we have $\widetilde{\nabla}_{X}^{\mathrm{C}} \widetilde{J} z=\widetilde{J} X$ as before. Thus, we find from (1.3) that

$$
\left\|A_{X}(\widetilde{J} z)\right\| \leqslant 1
$$

for each unit horizontal vector $X$ on $M$. Moreover, we know that the equality sign of (3.1) holds if and only if $\widetilde{J} X$ is a horizontal vector on $M$.

Now, suppose that the imbedding $\phi: M \rightarrow S^{2 n+1}$ satisfies the equality case of (1.6) identically. Then we obtain from (1.5), (1.6) and (3.1) that

$(\alpha) \quad M$ is imbedded as a minimal submanifold of $S^{2 n+1}$ and

$(\beta)$ the horizontal distribution $\mathcal{H}$ on $M$ is $\widetilde{J}$-invariant.

Since $\phi: M \rightarrow S^{2 n+1}$ carries the fibres of $\pi: M \rightarrow B$ to fibres of Hopf's fibration $\pi_{C}: S^{2 n+1} \rightarrow C P^{n}$, the imbedding $\phi$ gives rise to an isometric imbedding of $B$ into $C P^{n}$. Further, since the horizontal distribution $\mathcal{H}$ is $\widetilde{J}$-invariant, the imbedding of $B$ into $C P^{n}$ is a Kaehlerian. Thus, if we put

$$
M_{1}=\phi(M), \quad B_{1}=\pi_{C}\left(M_{1}\right), \quad \pi_{1}=\left.\pi_{C}\right|_{M_{1}},
$$

then $\pi: M \rightarrow B$ and $\pi_{1}: M_{1} \rightarrow B_{1}$ are equivalent and also $\phi$ is congruent to the inclusion map: $\iota_{C}: \pi_{1}^{1}\left(B_{1}\right) \rightarrow S^{n+1}$. Consequently, we obtain statement (i).

Next, assume that $M$ admits an isometric imbedding $\phi: M \rightarrow S^{4 k+3}$ which carries the fibres of $\pi$ to fibres of $\pi_{Q}: S^{4 k+3} \rightarrow Q P^{k}$.

Let $J_{1}, J_{2}, J_{3}$ denote the canonical almost complex structures on $\mathbb{Q}^{k+1}$ induced from the quaternionic structure of $\mathbb{Q}^{k+1}$. Then $J_{1}, J_{2}, J_{3}$ are parallel with respect to the flat Levi-Civita connection of $\mathbb{Q}^{k+1}$, that is, we have $\nabla^{\mathbb{Q}} J_{\alpha}=0$ for $\alpha=1,2,3$.

Let $z$ denote the position vector field of $M$ in $\mathbb{Q}^{k+1}$. Then $J_{1} z, J_{2} z, J_{3} z$ are unit vertical vector fields on $M$. For any horizontal vector $X$ of $M$, we have

$$
\tilde{\nabla}_{X}^{Q} J_{\alpha} z=J_{\alpha} X, \quad \alpha=1,2,3 .
$$

Thus, for any unit horizontal vector $X$, we obtain from (1.3) that

$$
\left\|A_{X}\left(J_{\alpha} z\right)\right\| \leqslant 1, \alpha=1,2,3 .
$$

We also know that the equality sign of (3.3) holds for each $\alpha \in\{1,2,3\}$ if and only if each $J_{\alpha} X$ is a horizontal vector on $M$.

Now, suppose that $\phi: M \rightarrow S^{4 k+3}$ satisfies the equality case of (1.6) identically. Then it follows from (1.5), (1.6) and (3.3) that $M$ is imbedded as a minimal submanifold of $S^{4 k+3}$; moreover, the horizontal distribution $\mathcal{H}$ on $M$ is invariant under $J_{1}, J_{2}$ and $J_{3}$.

Since $\phi: M \rightarrow S^{4 k+3}$ carries the fibres of $\pi: M \rightarrow B$ to fibres of $\pi_{Q}: S^{4 k+3} \rightarrow Q P^{k}$, the imbedding $\phi$ gives rise to an isometric imbedding of $B$ in $Q P^{k}$. Because the horizontal 
distribution $\mathcal{H}$ is invariant under $J_{1}, J_{2}, J_{3}, B$ must be imbedded in $Q P^{n}$ as an invariant submanifold. Hence, after applying Lemma 4 of $[3]$ we conclude that $B$ is imbedded as an open portion of a totally geodesic $Q P^{\ell} \subset Q P^{k}$. Therefore, if we put $B_{2}=\phi(B)$, $M_{2}=\phi(M)$ and $\pi_{2}=\iota_{Q} / M_{2}$, then we obtain statement (ii).

\section{Proof of Theorem 3.}

Let $\pi: M \rightarrow B$ be a Riemannian submersion with totally geodesic fibres. Denote by $\nabla$ the Levi-Civita connection of $M$ and by $R$ the Riemann curvature tensor of $M$. Then we have

$$
R(E, F)=\left[\nabla_{E}, \nabla_{F}\right]-\nabla_{[E, F]}
$$

for vector fields $E, F$ tangent to $M$.

Assume that $M$ admits an isometric immersion $\phi: M \rightarrow S^{N}$ which carries the fibres of $\pi$ to totally geodesic submanifolds of $S^{N}$. Then the second fundamental form $h$ of $M$ in $S^{N}$ satisfies

$$
h(V, W)=0
$$

for vertical vectors $V, W$ on $M$.

From the equation of Gauss we know that the sectional curvature function $K$ of $M$ satisfies (see, for instance, [2])

$$
K(X \wedge V)=1+\langle h(X, X), h(V, V)\rangle-\|h(X, V)\|^{2}
$$

for unit horizontal vector $X$ and unit vertical vector $V$ on $M$. Hence, from (4.1) and (4.2) we find

$$
K(X \wedge V) \leqslant 1
$$

for each unit horizontal vector $X$ and unit vertical vector $V$. It is easy to see that the equality sign of (4.3) holds if and only if we have $h(X, V)=0$.

On the other hand, since the submersion $\pi$ has totally geodesic fibres, $K$ satisfies (see [11, page 465])

$$
\left\|A_{X} V\right\|^{2}=K(X \wedge V) \leqslant 1
$$

for every unit horizontal vector $X$ and unit vertical vector $V$. Thus, by applying (1.5), (4.3) and (4.4), we obtain

$$
\breve{A}_{\pi} \leqslant b(m-b), \quad b=\operatorname{dim} B, \quad m=\operatorname{dim} M,
$$

with equality holding if and only if $M$ is mixed-totally geodesic in $S^{N}$, that is, $h(X, V)=0$ holds for any unit horizontal vector $X$ and unit vertical vector $V$ on $M$. Therefore, the equality sign of (1.6) holds implies that

$$
\breve{A}_{\pi}=b(m-b), \quad H=0 .
$$


Hence, $M$ is immersed as a minimal mixed-totally geodesic submanifold of $S^{N}$. The converse is easy to verify. This gives statement (2).

Next, assume that $\operatorname{dim} M=2$. Then (4.6) implies that the Gaussian curvature $G$ of $M$ satisfying $G=1$ and $M$ is immersed as a minimal surface in $S^{N}$ via $\phi$. From these we conclude that $M$ is totally geodesic in $S^{N}$. Hence, $M$ is an open portion of a unit 2-sphere $S^{2}$.

Let $e_{1}$ be a unit vertical vector field and $e_{2}$ be a unit horizontal vector field on $M$. Then, it follows from the totally geodesy of fibres of $\pi$ that $\nabla_{e_{1}} e_{1}=0$. On the other hand, it follows from (1.2) and (4.6) that we also have $\nabla_{e_{2}} e_{1}= \pm e_{2}$. By applying these we obtain $G=\left\langle R\left(e_{2}, e_{1}\right) e_{1}, e_{2}\right\rangle=-1$ which is a contradiction. Therefore, we obtain statement (1) of Theorem 3 .

\section{Proof of Theorem 4.}

Obviously, $S^{3}$ admits the Riemannian submersion $\pi_{C}: S^{3} \rightarrow C P^{1}$ with totally geodesic fibres and the inclusion map $S^{3} \subset S^{4}$ satisfies the equality case of (1.6) identically.

Now, assume that $M^{3}$ is a Riemannian 3-manifold and $\pi: M^{3} \rightarrow B$ is a Riemannian submersion with totally geodesic fibres. Suppose that $M^{3}$ admits a non-totally geodesic isometric immersion $\phi: M^{3} \rightarrow S^{4}$ which carries fibres to totally geodesic submanifolds and which satisfies the equality case of (1.6) identically. Then, according to Theorem 3, $M^{3}$ is immersed as a minimal mixed-totally geodesic hypersurface of $S^{4}$.

CASE (i). $\operatorname{dim} B=1$. In this case, the fibres of $\pi$ are 2-dimensional and the fibres are immersed as totally geodesic surfaces in $S^{4}$. Thus, by applying the mixed-totally geodesy and minimality of $\phi$, we know that $\phi$ is totally geodesic. This is a contradiction.

CASE (ii). $\operatorname{dim} B=2$. In this case, the fibres of $\pi$ are carries to great circles in $S^{4}$ by $\phi$. Hence, the second fundamental form $h$ of $\phi$ satisfies $h(V, V)=0$ for any vertical vector $V$. So, by applying the mixed-totally geodesy and minimality of $\phi$, we may choose $e_{1}$ to be a unit vertical vector field and $e_{2}, e_{3}$ to be orthonormal horizontal vector fields so that $e_{1}, e_{2}, e_{3}$ diagonalise the shape operator of $M$ in $S^{4}$. In this way we have

$$
\begin{aligned}
& h\left(e_{1}, e_{1}\right)=h\left(e_{1}, e_{2}\right)=h\left(e_{1}, e_{2}\right)=h\left(e_{2}, e_{3}\right)=0, \\
& h\left(e_{2}, e_{2}\right)=-h\left(e_{3}, e_{3}\right)=\lambda e_{4}
\end{aligned}
$$

for some function $\lambda \neq 0$, where $e_{4}$ is a unit normal vector field of $M^{3}$ in $S^{4}$.

Let $\nabla$ be the Levi-Civita connection of $M^{3}$ and put

$$
\nabla_{X} e_{i}=\sum_{j=1}^{3} \omega_{i}^{j}(X) e_{j},
$$


Since the submersion has totally geodesic fibres, we have

$$
\nabla_{e_{1}} e_{1}=0
$$

By applying (5.1)-(5.3) and the equation of Codazzi, we find

$$
\begin{gathered}
e_{1} \lambda=0, \quad e_{2} \lambda=2 \lambda \omega_{3}^{2}\left(e_{3}\right), \quad e_{3} \lambda=2 \lambda \omega_{2}^{3}\left(e_{3}\right), \\
\omega_{1}^{3}\left(e_{2}\right)=\omega_{2}^{1}\left(e_{3}\right)=2 \omega_{2}^{3}\left(e_{1}\right) .
\end{gathered}
$$

Because the integrability tensor $A$ satisfies the alternation property (1.4) for horizontal vector fields $X, Y$, we obtain

$$
\left\langle\nabla_{e_{i}} e_{1}, e_{i}\right\rangle=-\left\langle\nabla_{e_{i}} e_{i}, e_{1}\right\rangle=-\left\langle A_{e_{i}} e_{i}, e_{1}\right\rangle=0, \quad i=2,3 .
$$

Since the inequality (1.6) is an equality, we obtain from (4.4) and (4.6) that

$$
\left\|A_{e_{2}} e_{1}\right\|=\left\|A_{e_{3}} e_{1}\right\|=1 .
$$

Thus, by applying (5.4) and (5.5), we find

$$
\nabla_{e_{2}} e_{1}= \pm e_{3}, \quad \nabla_{e_{3}} e_{1}=\mp e_{2}
$$

Without loss of generality, we may assume that

$$
\nabla_{e_{2}} e_{1}=e_{3}, \quad \nabla_{e_{3}} e_{1}=-e_{2}
$$

Hence, by combining (5.3), (5.4) and (5.6), we obtain

$$
\begin{array}{lll}
\nabla_{e_{1}} e_{1}=0, & \nabla_{e_{2}} e_{1}=e_{3}, & \nabla_{e_{3}} e_{1}=-e_{2}, \\
\nabla_{e_{1}} e_{2}=\frac{e_{3}}{2}, & \nabla_{e_{2}} e_{2}=\frac{1}{2} e_{3}(\ln \lambda) e_{3}, & \nabla_{e_{3}} e_{2}=e_{1}-\frac{1}{2} e_{2}(\ln \lambda) e_{3}, \\
\nabla_{e_{1}} e_{3}=-\frac{e_{2}}{2}, & \nabla_{e_{2}} e_{3}=-e_{1}-\frac{1}{2} e_{3}(\ln \lambda) e_{2}, & \nabla_{e_{3}} e_{3}=\frac{1}{2} e_{2}(\ln \lambda) e_{2} .
\end{array}
$$

It follows from $e_{1} \lambda=0,(5.1),(5.7)$, and the equation of Gauss that the Riemann curvature tensor $R$ of $M^{3}$ satisfies

$$
0=4\left\langle R\left(e_{1}, e_{2}\right) e_{3}, e_{2}\right\rangle=e_{2}(\ln \lambda)
$$

Similarly, we find

$$
0=4\left\langle R\left(e_{1}, e_{3}\right) e_{3}, e_{2}\right\rangle=e_{3}(\ln \lambda) .
$$

It follows from $e_{1} \lambda=0,(5.8)$ and (5.9) that $\lambda$ is constant. Hence, $M^{3}$ is immersed as a minimal isoparametric hypersurface in $S^{4}$.

Finally, by applying (5.1), (5.7), the equation of Gauss, and the constancy of $\lambda$, we obtain

$$
1-\lambda^{2}=\left\langle R\left(e_{2}, e_{3}\right) e_{3}, e_{2}\right\rangle=-2 .
$$

Thus, we obtain $\lambda^{2}=3$. Consequently, $\phi: M^{3} \rightarrow S^{4}$ is congruent to the Cartan hypersurface in $S^{4}$. 


\section{An AdDitional RESUlt.}

Consider $S^{2 n+1}$ as the unit sphere in $\mathbb{C}^{n+1}$ as before. For each tangent vector field of $S^{2 n+1}$, let $\varphi(E)$ denote the tangential component of $\tilde{J} E$, where $\tilde{J}$ is the almost complex structure on $\mathbb{C}^{n+1}$. Then $\varphi$ is the endomorphism on Sasakian manifold $\left(S^{2 n+1}, g, \varphi, \xi, \eta\right)$ which satisfies

$$
\varphi^{2}=-I+\eta \otimes \xi,
$$

where $I$ is the identity map, $\xi=\widetilde{J}_{z}$ is the characteristic vector field, and $\eta$ is the dual 1-form of $\xi$ (see [1, page 33]).

Let $B$ be a submanifold of $C P^{n}$. Then the natural projection $\pi: \pi_{C}^{-1}(B) \rightarrow B$ is a Riemannian submersion with totally geodesic fibres. Let $\iota: \pi_{C}^{-1}(B) \rightarrow S^{2 n+1}$ denote the inclusion map.

In views of Theorem 1 and Theorem 3 we give the following.

Proposition 5. The immersion $\iota: \pi_{C}^{-1}(B) \rightarrow S^{2 n+1}$ is mixed-totally geodesic if and only if the horizontal distribution of $\pi: \pi_{C}^{-1}(B) \rightarrow B$ is $\varphi$-invariant.

Proof: Let $z$ denote the unit outward normal of $S^{2 n+1} \subset \mathbb{C}^{m+1}$. Then, for any horizontal vector $X$ of $\pi: M \rightarrow B$, we have

$$
\tilde{\nabla}_{X}^{\mathcal{C}}(\tilde{J} z)=\tilde{J} \widetilde{\nabla}_{X} z=\tilde{J} X
$$

where $\widetilde{\nabla}^{\mathbb{C}}$ and $\widetilde{J}$ are the Levi-Civita connection and the almost complex structure of $\mathbb{C}^{m+1}$. Since $V=\tilde{J} z$ is a unit vertical vector field of $\pi,(6.1)$ implies that the second fundamental form $h$ of $M$ in $S^{2 n+1}$ satisfies

$$
h(X, V)=F X,
$$

where $F X$ is the normal component of $\widetilde{J} X$ in $S^{2 n+1}$.

Since $\widetilde{J} X$ is perpendicular to $z$ and $\widetilde{J} z,(6.2)$ implies that $\iota: \pi_{C}^{-1}(B) \rightarrow S^{2 n+1}$ is mixed-totally geodesic if and only if the horizontal distribution $\mathcal{H}$ of $\pi: \pi_{C}^{-1}(B) \rightarrow B$ is $\varphi$-invariant.

REMARK 1. Similar result holds if $B$ is a submanifold of $Q P^{m}, \iota: \pi_{Q}^{-1}(B) \rightarrow S^{4 m+3}$ is the restriction of $\pi_{Q}$ to $\pi_{Q}^{-1}(B)$, and if $\varphi$ were replaced by $\varphi_{1}, \varphi_{2}, \varphi_{3}$ (induced from $J_{1}, J_{2}, J_{3}$ ) from the 3-Sasakian structures on $S^{4 m+3}$ (for 3-Sasakian structures, see, for instance, [1, pp. 215-216]).

\section{REFERENCES}

[1] D.E. Blair, Riemannian geometry of contact and symplectic manifolds (Birkhäuser Boston, Inc., Boston, MA, 2002).

[2] B.Y. Chen, Geometry of submanifolds (Mercel Dekker, New York, 1973).

[3] B.Y. Chen, 'Totally umbilical submanifolds of quaternion-space-forms', J. Austral. Math. Soc. Ser. A 26 (1978), 154-162. 
[4] B.Y. Chen, 'Some pinching and classification theorems for minimal submanifolds', Arch. Math. 60 (1993), 568-578.

[5] B.Y. Chen, 'Some new obstructions to minimal and Lagrangian isometric immersions', Japan. J. Math. 26 (2000), 105-127.

[6] B.Y. Chen, 'Riemannian submersions, minimal immersions and cohomology class', (submitted).

[7] R.H. Escobales, Jr., 'Riemannian submersions with totally geodesic fibers', J. Differential Geom. 10 (1975), 253-276.

[8] R.H. Escobales, Jr., 'Riemannian submersions from complex projective space', J. Differential Geom. 13 (1978), 93-107.

[9] W.Y. Hsiang and H.B. Lawson, Jr., 'Minimal submanifolds of low cohomogeneity', J. Differential Geom. 5 (1971), 1-38.

[10] T. Nagano, 'On fibred Riemann manifolds', Sci. Papers College Gen. Ed. Univ. Tokyo 10 (1960), 17-27.

[11] B. O'Neill, 'The fundamental equations of a submersion', Michigan Math. J. 13 (1966), 459-469.

Department of Mathematics

Michigan State University

East Lansing, MI 48824-1027

United States of America

e-mail: bychen@math.msu.edu 